\title{
Représentations envers les difficultés de la grammaire française - cas de futurs philologues polonais
}

\author{
Maciej Smuk \\ Université de Varsovie, Institut d'Études Romanes, Département de Didactique des \\ Langues Romanes \\ m.smuk@uw.edu.pl
}

\begin{abstract}
Résumé. L'objectif du présent article est d'analyser les représentations se rapportant aux difficultés de la grammaire française des étudiants en philologie romane (études de langue et de culture françaises). Après avoir exposé les principaux traits et les enjeux de la notion de représentation mentale, nous présenterons et discuterons les résultats de l'étude empirique réalisée auprès de 104 étudiants polonais, faisant leurs études à l'Institut d'Études Romanes de l'Université de Varsovie. Durant la première étape de la recherche, nous avons soumis à une analyse leur conscience terminologique, celle-ci pouvant prouver une plus grande conscience du fonctionnement de la langue. Pendant la deuxième phase, afin d'accéder aux représentations des répondants, nous leur avons demandé de se prononcer librement sur le degré de difficulté de divers aspects de la grammaire française et d'expliciter les raisons de tel ou tel sentiment. Nous avons mis l'accent, tant quant au contenu qu'à la forme, sur la perspective qualitative et subjective. Notre recherche avait un caractère introspectif, puisant dans une approche herméneutique et phénoménologique.
\end{abstract}

\begin{abstract}
Representations of difficulties of French grammar - the case of Polish future philologists. The purpose of this article is to analyse representations related to the difficulties of French grammar regarding students in French philology (studies of French language and culture). After presenting the main features and issues of the notion of mental representation, we will present and discuss the results of an empirical study conducted with 104 Polish students, studying at the Warsaw University's Institute of French Studies. During the first stage of the research, we subjected to an analysis their terminological awareness, which can prove a greater awareness of the functioning of the language. During the second phase, in order to access the representations of the respondents, we asked them to freely decide on the degree of difficulty of various aspects of French grammar and to explain the reasons for this or that feeling. We emphasised, both in terms of content and form, on the qualitative and subjective perspective. Our research was introspective, drawing on a hermeneutic and phenomenological approach.
\end{abstract}




\section{En guise d'introduction}

Toute langue est une forme de représentation du monde - le concept de représentation est bien connu des linguistes. Mais du point de vue de la recherche sur la cognition, c'est déjà en 1818, par la constatation "Le monde est ma représentation », qu'A. Schopenhauer a attiré l'attention, dans «Le monde comme volonté et comme représentation », sur cette perspective : tout objet du monde peut être interprété différemment, en fonction des caractéristiques de celle ou celui qui l'interprète ; dans ce sens, la réalité ne serait objective qu'en apparence. Dans l'ouvrage en question, A. Schopenhauer explicite son idée de manière figurative : "Cette proposition est une vérité pour tout être vivant et pensant, bien que, chez l'homme seul, elle arrive à se transformer en connaissance abstraite et réfléchie (...). Il possède (...) l'entière certitude de ne connaître ni un soleil ni une terre, mais seulement un œil qui voit ce soleil, une main qui touche cette terre ; il sait, en un mot, que le monde dont il est entouré n'existe que comme représentation dans son rapport avec un être percevant, qui est l'homme lui-même » (Schopenhauer, 1818/2004). Mais c'est seulement avec la théorie des représentations sociales, élaborée par S. Moscovici (1961), faisant référence au concept de représentations collectives d'É. Durkheim (1898), que cette notion a gagné en popularité dans les sciences humaines. Les représentations sociales désignent les manières, différentes selon la culture, de construire et d'organiser les connaissances dites objectives. Elles se greffent aux aspects cognitifs, mais aussi aux croyances, aux idéologies, aux valeurs ou aux pratiques fréquentes propres à un groupe social donné. Néanmoins, les représentations sont dynamiques et elles évoluent au rythme de l'évolution de ces groupes - c'est, par exemple, une des raisons pour lesquelles le rapport à l'histoire de sa culture peut changer au fil du temps.

Les représentations mentales individuelles, conceptuelles et procédurales, se forgent souvent sous l'effet des représentations sociales, entre autres suite à la scolarisation. Dans ce processus, leur fonction est d'une valeur majeure, puisqu'elles influent directement sur l'attention accordée à divers sujets et aspects, sur leur conceptualisation ainsi que sur la perception du degré de leur difficulté (les représentations envers la difficulté jouent, maintes fois, un rôle décisif dans la motivation pour entamer l'apprentissage d'un nouveau sujet). Les enjeux ébauchés ci-dessus expliquent pourquoi les représentations suscitent un vif intérêt auprès de spécialistes de nombreux domaines : psychologie, psychothérapie, pédagogie, sociologie, ethnographie, didactique, etc.

Pour ce qui est du présent article, l'objectif principal que nous nous étions fixé était d'étudier les représentations des apprenants envers les difficultés de la grammaire française. Pour ce faire, nous avons interrogé des étudiants polonais en philologie romane (études de langue et de culture françaises) de l'Université de Varsovie. Examiner leurs représentations devait expliquer les raisons de divers niveaux de difficultés face à divers aspects, aboutir à une meilleure compréhension des mécanismes activés durant l'apprentissage de la grammaire et pourrait donc avoir, comme conséquence, l'optimisation des démarches entreprises par les enseignants pendant les cours de langue française/grammaire.

\section{Représentations mentales des apprenants}

\subsection{Définitions et particularités}

Comme nous l'avons signalé, la notion de représentation, plus exactement de représentation mentale, prend sa source dans des travaux relevant de la psychologie sociale et cognitive, et d'après une métaphore popularisée, elle signifie «un déjà là » conceptuel. Les représentations assument donc le rôle de variables intervenantes, donc celles qui interviennent entre les stimuli, par exemple une activité de grammaire à réaliser, et les 
conduites, soit la manière de la réaliser. L'attitude, terme apparenté à la représentation, désigne une disposition interne latente par rapport à divers phénomènes, trouvant son reflet dans les comportements. Du point de vue de sa structure, l'esprit se compose de multiples représentations liées les unes aux autres - elles reflètent des images plus globales (p. ex. "La grammaire française est facile, difficile, logique, alogique, ... ») ou bien elles sont traitées comme les correspondants mentaux des concepts et se rapportent à leurs composantes particulières (p. ex. " Le subjonctif est très difficile, le mode le plus utile, plus difficile que le conditionnel, ...»). Du point de vue des processus mis en œuvre, l'esprit manie les représentations, à savoir les interprète, les ajoute aux précédentes et les utilise pour créer de nouvelles représentations. Les modèles mentaux discutés se caractérisent par leur organisation et cohérence internes spécifiques : toute nouvelle structure se greffe aux structures cognitives déjà acquises, mais - soulignons-le - les aspects cognitifs et affectifs (aspirations, croyances, préférences, stéréotypes, etc.) sont inséparables ; en outre, de même que les représentations sociales, les représentations individuelles peuvent ne point correspondre aux connaissances scientifiques et rationnelles. En fait, les représentations sont des interprétations, souvent non conscientisées, qui filtrent de nouveaux acquis (p. ex. la représentation d'un problème grammatical influence la façon d'aborder l'autre) et, contrairement aux premières apparences, elles n'équivalent pas aux concepts. Selon C. Meyer (2001), si la représentation en tant qu'image mentale est active, opératoire et toujours construite par l'individu, le concept « (...) ne possède pas de caractère figuratif. Il est (...) composé d'unités élémentaires, supports de discrimination : les traits sémantiques. Il est une représentation générale et abstraite (...), un objet logique stable (...) ». Dans la littérature sur le sujet, au moins quelques types de représentations sont distingués : plus et moins immuables, permanentes et momentanées, conscientes et inconscientes, actives et emmagasinées.

Les représentations liées aux objets de l'enseignement/apprentissage s'ancrent dans les expériences scolaires antérieures et, ne l'oublions pas, les enseignants, eux aussi, lancent leurs représentations à propos des sujets en question au moment de leur présentation. Même si elles sont évolutives et susceptibles d'être modifiées, entre autres (peut-être surtout ?) par les enseignants, elles constituent quelquefois une sérieuse entrave à l'acquisition de nouvelles connaissances. Deux scénarios néfastes peuvent être envisagés: soit les représentations «préexistantes » sont profondément internalisées, mais elles ne sont pas conscientisées, soit une longue distance les sépare de nouvelles représentations en cours de formation. Comme nous venons de le dire, les représentations peuvent s'appuyer sur des informations objectives, mais, dans la plupart des cas, elles se fondent aussi sur des expériences particulières, voire sur des stéréotypes et des craintes individuelles portant sur l'objet concerné (p. ex. «La grammaire française est particulièrement compliquée. Bien maîtriser le subjonctif est infaisable. Les pronoms personnels en français s'utilisent intuitivement - il est donc peu utile d'apprendre les règles de leur emploi. »). Les représentations deviennent des ingrédients importants des théories dites subjectives, plus complexes et plus difficiles à cerner.

\subsection{Enjeux et fonctions}

Deux grands enjeux des représentations s'attachent à leur nature même : les unes font partie de la mémoire à long terme et peuvent être qualifiées d'interprétations plus générales des objets, les autres sont mises en œuvre par la mémoire de travail durant l'exécution des tâches et jouent ainsi le rôle de "structures transitoires de compréhension » (Castelotti, 2001) ; elles influent donc également sur la rapidité de décision et de jugement, mais pour la même raison, elles sont aussi à l'origine de certaines erreurs (Meyer, 2001). Les deux orientent la manière de procéder et déterminent des anticipations par rapport aux objets, mais seulement les secondes, dénommées « représentations procédurales » ou «modèles mentaux procéduraux », sont des savoir-faire. Les représentations font référence aux 
connaissances déclaratives transformées en procédurales par le biais de la mémoire sémantique et résultent aussi des expériences encodées par la mémoire épisodique.

Trois fonctions essentielles peuvent être attribuées aux représentations dans le contexte de l'enseignement/apprentissage (www.lmg.ulg.ac.be). Premièrement, l'apprenant, par l'intermédiaire de leur verbalisation, est en mesure de les conscientiser et, au besoin, il peut les confronter à celles des autres. Il est également désirable qu'il suive leur évolution au fil du temps. Deuxièmement, c'est surtout l'enseignant-chercheur qui dispose d'une palette de possibilités: comprendre les perceptions des apprenants, effectuer les comparaisons interindividuelles et établir les représentations les plus fréquentes, comparer les représentations des apprenants venant de diverses cultures langagières/éducatives. Enfin, lors des cours, l'enseignant et les apprenants peuvent confronter leurs représentations, les mettre en question, expliciter des réticences et, donc, apporter des modifications nécessaires et remédier, le cas échéant, à quelques-unes des barrières enracinées dans des représentations non pertinentes; nous recommanderions vivement ces démarches avant de procéder à l'analyse de tels ou tels sujets, surtout s'ils diffèrent considérablement de ceux de la langue maternelle. À notre avis, il est aussi souhaitable de distinguer les représentations individuelles des représentations sociales, donc partagées par des groupes plus vastes d'apprenants.

\section{3 Étude des représentations en matière d'acquisition des langues}

Dans leur guide pour l'élaboration des politiques linguistiques éducatives, V. Castellotti et D. Moore (2002) rappellent à juste titre: «Pour la linguistique de l'acquisition, les représentations constituent un élément structurant du processus d'appropriation langagière. Les représentations sur la langue maternelle, sur la langue à apprendre, et sur leurs différences sont liées à certaines stratégies d'apprentissage chez les apprenants qui se construisent une représentation de la distance interlinguistique séparant le système de leur langue de celui de la langue à apprendre ». Les recherches portant sur les représentations sont abondantes dans le domaine de l'acquisition des langues, mais elles concernent avant tout les représentations de la langue apprise (son statut, ses usages, etc.), l'importance de différents sous-systèmes langagiers, le parcours de l'apprentissage ou bien les milieux parlant telle ou telle langue. Les études empiriques à caractère introspectif, y compris les travaux de confrontation entre ses productions et les verbalisations (jugement de grammaticalité, définition de règles, etc.), initiées dans les années soixante-dix par S. P. Corder (1973), montrent des divergences significatives entre « (...) ce que l'apprenant fait et ce qu'il croit faire, entre les règles dont il parle et celles qu'il applique réellement » (Vasseur et Arditty, 1996). Sans doute, l'image mentale du degré de complexité et de difficulté de l'objet et de la tâche à effectuer met ses empreintes sur la façon dont ils seront abordés.

\section{1 Étude empirique}

\subsubsection{Objectif et questions de recherche}

Notre recherche avait un caractère exploratoire. Son objectif primordial était de faire apparaître les représentations envers les difficultés de la grammaire française. Nous avons formulé cinq questions de recherche s'articulant à l'objectif de départ :

1. Quelle est la conscience terminologique des apprenants quant aux termes relevant du domaine de la grammaire?

2. Quels aspects de la grammaire française passent, aux yeux des apprenants, pour les plus difficiles?

3. Quels sont les fondements, cognitifs et affectifs, de ces représentations? 
4. Serait-il possible d'établir une hiérarchie des difficultés du point de vue des deux groupes de répondants participant à la recherche (étudiants moins et plus avancés, cf. 3.1.2)?

5. Existe-t-il donc des dissemblances significatives entre les deux groupes de répondants et, si oui, où se manifestent-elles davantage?

Afin de répondre à ces interrogations, nous avons recouru au recueil de données quantitatives et qualitatives sur les représentations concernant le degré de difficulté des aspects grammaticaux que ressentent les apprenants en FLE. Se focaliser sur cette perspective nous a paru beaucoup plus pertinent que de se baser sur un recueil de représentations sur des aspects grammaticaux tels quels (p. ex. «Terminez la phrase : Les pronoms personnels sont pour moi ... » ou « Racontez vos expériences d'apprentissage des pronoms personnels »). D'après nos expériences préalables, des consignes analogiques peuvent déboucher sur la description de procédures appliquées par les enseignants ou la reproduction d'idées reçues (p. ex. «Les pronoms personnels sont pour moi difficiles, mais je sais qu'il faut les maîtriser $»)$.

\subsubsection{Contexte de l'étude et échantillon}

Notre étude empirique a été effectuée auprès d'étudiants en philologie romane (études de langue et de culture françaises) de l'Institut d'Études Romanes de l'Université de Varsovie. L'Institut appartient à la Faculté de Langues Modernes où sont formés des spécialistes, interprètes et enseignants essentiellement, en huit langues : allemand, anglais, espagnol, finnois, français, hongrois, italien, portugais. Au total, environ 2500 personnes y font leurs études. L'Institut d'Études Romanes offre trois parcours :

- études du premier cycle (licence) pour les candidats sans maîtrise de la langue française ;

- études du premier cycle (licence) pour les candidats maîtrisant la langue française au niveau B1 du Cadre européen commun de référence pour les langues ;

- études du second cycle (masters 1 et 2) pour les candidats maîtrisant la langue française au niveau $\mathrm{C} 1$ du Cadre européen commun de référence pour les langues.

Les étudiants du premier cycle en philologie romane suivent de 2348 à 2558 heures de cours, dont 1020 sont destinées aux cours de langue française pour les débutants et $870-$ pour les avancés. Les étudiants du second cycle réalisent au total de 780 à 840 heures de cours, dont 180 sont prévues pour les cours de langue. Outre les cours de français pratique, les étudiants suivent des cours obligatoires dans les domaines suivants : analyse de textes littéraires, analyse syntaxique, francophonie, grammaire descriptive de la langue française, histoire de la littérature française, histoire et culture de France, langue latine, linguistique générale, méthodologie de la recherche en didactique des langues/linguistique/littérature, philosophie française, phonétique de la langue française, didactique des langues, divisée en quelques modules thématiques, séminaires thématiques optionnels, choisis en fonction des intérêts ou des besoins, etc. La plupart des cours sont entièrement menés en français.

Les cours de français pratique sont répartis en plusieurs volets : enseignement intégré des compétences langagières, entraînement stratégique à l'apprentissage, expression orale et écrite, grammaire appliquée et phonétique appliquée. À ceux-ci s'ajoutent des volets thématiques: actualités françaises et francophones, traduction écrite / orale / cinématographique, français des affaires, etc. Les cours de grammaire appliquée, comptant 210 heures, doivent permettre d'approfondir les connaissances acquises au lycée et de répondre aux problèmes soulevés lors des cours d'expression orale et écrite.

En somme, nous avons interrogé 104 personnes : pour comparer aisément les données quantitatives, 52 étudiants de la première année maîtrisant le français au niveau B1 (désormais groupe $\mathrm{A}$ ) et 52 étudiants de la troisième année en licence et du master, donc ceux qui ont déjà suivi au moins quatre semestres d'études de langue française (désormais groupe B). L'échantillon est constitué de 88 étudiantes et 16 étudiants, ce qui reflète la 
démographie de la filière. Leur âge moyen est de 20 ans. La recherche a été effectuée au semestre d'hiver 2017/2018.

\subsubsection{Démarches et procédures}

Avant de nous lancer dans l'étude des représentations portant sur les difficultés de la grammaire française, nous avons décidé de déterminer le degré de conscience dite terminologique des répondants. Cette première étape s'est imposée comme particulièrement appropriée pour deux raisons. D'un côté, la reconnaissance des termes correspondant aux parties de la grammaire semble témoigner d'une plus grande conscience du fonctionnement de la langue (Cuq et Gruca, 2005). D'un autre côté, nous avons admis que la tâche consistant à reconnaître les termes aurait une fonction d'initialisation : elle devait permettre d'activer des connaissances déclaratives dans le domaine en question et d'évoquer déjà certaines représentations quant aux aspects grammaticaux donnés. Ceci devait également faciliter aux répondants d'accéder aux étapes postérieures de la recherche. Pour définir ladite conscience terminologique, deux activités s'enchaînant ont été proposées aux répondants. Initialement, ils ont été invités à donner un exemple des parties du discours suivantes : adjectif démonstratif, adjectif indéfini, adjectif numéral cardinal, adjectif numéral ordinal, adjectif possessif, adjectif qualificatif, adverbe, article contracté, article défini, article indéfini, article partitif, conjonction, préposition, pronom démonstratif, pronom indéfini, pronom personnel complément d'objet direct (COD), pronom personnel complément d'objet indirect (COI), pronom personnel sujet, pronom personnel tonique, pronom possessif, pronom relatif simple, pronom relatif composé, substantif. Après avoir accompli la première activité, les répondants ont été priés d'associer les termes grammaticaux donnés ci-dessus aux mots qui suivent: troisième, trois, hier, la veille, une, celles, parmi, cet, aux, le, chacun, tu, du, chaque, timide, toi, le mien, dont, leur, mon, me, et, auxquelles. Quelquefois, un même mot appartenait à plus d'une catégorie. Les résultats obtenus seront donnés dans la section 3.1.4.1.

Pendant la deuxième étape de l'étude empirique, nous avons demandé aux répondants d'évoquer trois aspects de la grammaire française engendrant pour eux le plus de difficultés dans le processus d'apprentissage et/ou de communication. Pour chaque aspect, ils devaient essayer d'expliquer, dans la langue de leur choix, les raisons de ces difficultés. Les résultats de cette partie de l'étude seront présentés dans la section 3.1.4.2.

Il découle de ce qui précède que nous avons eu recours, dans notre recherche, à la technique d'introspection qui se prête le mieux à l'étude des représentations. La production libre portant sur un sujet donné, surtout sa forme écrite, s'inscrivant dans une approche herméneutique et phénoménologique, favorise tout particulièrement la réflexion et l'ouverture. De plus, la verbalisation en soi est considérée par les spécialistes comme un excellent moyen de prise de conscience et d'autoévaluation. La perspective subjective, trouvant son reflet déjà au niveau des mots et des expressions utilisés pour décrire la réalité, constitue l'un des plus grands avantages de ladite technique de collecte de données, en particulier par rapport à celles de type purement quantitatif : « (...) les conclusions qui en découlent permettent de décortiquer les perceptions très subjectives des phénomènes et de comprendre les dépendances, parfois uniques en leur genre, se nouant entre ceux-ci » (Smuk, 2017).

\subsubsection{Analyse des résultats et discussion}

\subsubsection{Conscience terminologique}

Le but de la première étape de notre étude était d'identifier le degré de conscience terminologique des répondants. Nous leur avons demandé de donner un exemple pour chacune des 23 catégories grammaticales. Le choix de ces catégories avait été dicté par le programme du cursus de grammaire. Les résultats, présentés dans le tableau 1 dans l'ordre 
croissant, sont arrondis aux chiffres entiers supérieurs (chiffre après la virgule supérieur à $0,50)$.

Tableau 1. Conscience terminologique : " donnez un exemple de chaque catégorie ».

\begin{tabular}{|l|c|c|c|}
\hline \multirow{2}{*}{ Catégorie grammaticale } & \multicolumn{3}{|c|}{ Nombre de réponses correctes } \\
\cline { 2 - 4 } & $\begin{array}{c}\text { Total } \\
\text { (sur 104) }\end{array}$ & $\begin{array}{c}\text { Groupe A } \\
\text { (sur 52) }\end{array}$ & $\begin{array}{c}\text { Groupe B } \\
(\text { sur 52) }\end{array}$ \\
\hline 1. Pronom indéfini & $23(22 \%)$ & $1(2 \%)$ & $22(41 \%)$ \\
\hline 2. Pronom possessif & $31(30 \%)$ & $6(11 \%)$ & $25(46 \%)$ \\
\hline 3. Adjectif indéfini & $33(32 \%)$ & $0(0 \%)$ & $33(61 \%)$ \\
\hline 4. Pronom démonstratif & $38(37 \%)$ & $12(22 \%)$ & $26(48 \%)$ \\
\hline 5. Pronom relatif simple & $51(49 \%)$ & $8(15 \%)$ & $43(80 \%)$ \\
\hline 6. Pronom relatif composé & $52(50 \%)$ & $4(7 \%)$ & $48(89 \%)$ \\
\hline 7a. Pronom personnel sujet & $58(56 \%)$ & $16(30 \%)$ & $42(78 \%)$ \\
\hline 7b. Pronom personnel tonique & $58(56 \%)$ & $18(33 \%)$ & $40(74 \%)$ \\
\hline 8a. Adjectif démonstratif & $62(60 \%)$ & $17(31 \%)$ & $45(83 \%)$ \\
\hline 8b. Article contracté & $62(60 \%)$ & $20(37 \%)$ & $42(78 \%)$ \\
\hline 9. Adjectif possessif & $69(66 \%)$ & $22(41 \%)$ & $47(87 \%)$ \\
\hline $\begin{array}{l}\text { 10. Pronom personnel complément } \\
\text { d'objet direct (COD) }\end{array}$ & $73(70 \%)$ & $27(50 \%)$ & $46(85 \%)$ \\
\hline 11. Article partitif & $74(71 \%)$ & $30(56 \%)$ & $44(81 \%)$ \\
\hline $\begin{array}{l}\text { 12. Pronom personnel complément } \\
\text { d'objet indirect (COI) }\end{array}$ & $79(76 \%)$ & $34(63 \%)$ & $45(83 \%)$ \\
\hline 13. Préposition & & & \\
\hline 14a. Adjectif numéral ordinal & $98(79 \%)$ & $31(57 \%)$ & $51(94 \%)$ \\
\hline 14b. Adverbe & $98(94 \%)$ & $46(85 \%)$ & $52(96 \%)$ \\
\hline 14c. Substantif & $98(94 \%)$ & $46(85 \%)$ & $52(96 \%)$ \\
\hline 15. Conjonction & $101(97 \%)$ & $50(93 \%)$ & $51(94 \%)$ \\
\hline 16a. Adjectif qualificatif & $102(98 \%)$ & $50(93 \%)$ & $52(96 \%)$ \\
\hline 16b. Article défini & $102(98 \%)$ & $50(93 \%)$ & $52(96 \%)$ \\
\hline 16c. Article indéfini & $102(98 \%)$ & $50(93 \%)$ & $52(96 \%)$ \\
\hline 17. Adjectif numéral cardinal & $103(99 \%)$ & $51(94 \%)$ & $52(96 \%)$ \\
\hline
\end{tabular}

D'une perspective quantitative, les résultats se présentent comme suit : sur 23 points au maximum, la moyenne pour le groupe A est de 12,26 points (53\% de réponses correctes), pour le groupe $\mathrm{B}-18,74$ points ( $81 \%$ de bonnes réponses). Dans le groupe $\mathrm{A}$, le meilleur résultat s'est élevé à 20 points; dans le groupe $\mathrm{B}$, neuf répondants ont obtenu le score maximal. Même si des écarts importants de résultat demeurent entre les deux groupes, les tendances ne diffèrent pas significativement. Dans les deux groupes, les résultats montrent que c'est l'identification des pronoms et adjectifs indéfinis qui engendre le plus de difficultés $(22 \%$ et $32 \%$ de réponses correctes $)$. Le problème concerne aussi l'identification des pronoms possessifs et démonstratifs $(30 \%$ et $37 \%$ de réponses correctes) ; parallèlement, les adjectifs possessifs et démonstratifs ne présentent pas les mêmes difficultés. Ce résultat global reflète les tendances observées dans les deux groupes - le moins de réponses correctes se rapportent aux dites catégories. Relativement peu de répondants ont été capables de donner un exemple des pronoms relatifs (49-50\%) et des pronoms personnels : sujet et tonique (56\%). Environ $40 \%$ des répondants ont commis une faute dans les adjectifs possessifs et démonstratifs ainsi que les articles contractés. Les cinq erreurs les plus fréquentes se rapportent à :

- la confusion des termes «adjectif» et «pronom », essentiellement pour ce qui est des démonstratifs, des indéfinis et des possessifs ; 
- la confusion de la préposition de avec les articles contractés et les articles partitifs (la préposition de est qualifiée d'article);

- l'utilisation du terme « article partitif» pour la construction d'un/d'une ;

- l'incapacité de donner un exemple des pronoms relatifs (simples et composés) ;

- la confusion des pronoms personnels sujets/toniques/COD/COI (p. ex. je/moi/me ; ils/elles/eux/elles/les/leur).

Selon toute probabilité, la majorité des erreurs sont liées à l'absence des catégories en question (adjectifs versus pronoms, divers déterminants du nom, pronoms toniques) dans la langue polonaise. Il est en revanche plus difficile d'expliquer les raisons pour lesquelles un certain nombre de fautes a été noté dans les catégories familières (prépositions, adverbes, substantifs) et existant dans la langue maternelle - on peut éventuellement les attribuer aux variables intervenantes occasionnelles (incompréhension des termes français, troubles de concentration).

\subsubsection{Représentations envers la grammaire française}

Mises à part les constatations très imprécises comme «En général, j’ai des difficultés avec toute la grammaire française » $(\mathrm{R} 37)$ ou - tout au contraire - « En général, c'est le truc pour mémoriser, alors la grammaire est facile » (R72), l'analyse des données d'un point de vue quantitatif permet de découvrir certaines tendances quant aux difficultés de la grammaire française. Le tableau 2 permet de les comparer.

Tableau 2. Aspects de la grammaire française les plus difficiles aux yeux des répondants.

\begin{tabular}{|l|c|c|}
\hline \multicolumn{1}{|c|}{ Aspects de la grammaire française } & \multicolumn{2}{c|}{ Nombre de répondants } \\
\cline { 2 - 3 } & $\begin{array}{c}\text { Groupe A } \\
\text { (sur 52) }\end{array}$ & $\begin{array}{c}\text { Groupe B } \\
\text { (sur 52) }\end{array}$ \\
\hline Accord du participe passé & $3(6 \%)$ & $12(23 \%)$ \\
\hline Accords en genre et en nombre & 1 & 4 \\
\hline Adjectif et participe présent & 0 & 1 \\
\hline Adjectif qualificatif (place de) & 1 & 0 \\
\hline Articles & $25(48 \%)$ & $33(63 \%)$ \\
\hline Articulateurs logiques, relations logiques & 0 & 8 \\
\hline C'est et il est & 1 & 0 \\
\hline Cela et ça & 1 & 0 \\
\hline Conditionnel & $7(13 \%)$ & $6(11 \%)$ \\
\hline Démonstratifs (adjectifs et pronoms) & 1 & 0 \\
\hline Discours indirect & 3 & 0 \\
\hline Fonctions des parties du discours & 0 & 2 \\
\hline Indéfinis (adjectifs et pronoms) & 0 & 2 \\
\hline Ne explétif & 1 & 0 \\
\hline Passif & $3(6 \%)$ & $2(4 \%)$ \\
\hline Pluriel des substantifs & 0 & 1 \\
\hline Prépositions & $6(11 \%)$ & $6(11 \%)$ \\
\hline Pronoms personnels, surtout COD/COI & $24(46 \%)$ & $4(8 \%)$ \\
\hline Pronoms relatifs & $4(8 \%)$ & $9(17 \%)$ \\
\hline Subjonctif & $33(63 \%)$ & $18(35 \%)$ \\
\hline Syntaxe/structure de la phrase & $2(4 \%)$ & $8(15 \%)$ \\
\hline Temps de l'indicatif & $24(46 \%)$ & $14(27 \%)$ \\
\hline Verbes irréguliers : conjugaison & 2 & 1 \\
\hline
\end{tabular}


Dans le groupe A, au palmarès des sujets considérés comme difficiles figure le subjonctif. Néanmoins, il a été aussi assez souvent évoqué par les répondants du groupe B. Le premier obstacle, à caractère essentiellement psychologique, selon nous, réside dans le fait qu'un mode tel que le subjonctif n'existe pas dans la langue polonaise. Tel est l'avis de beaucoup d'étudiants, p. ex. «[le subjonctif] est difficile tout simplement, car il n'existe pas en polonais » (R74). Parallèlement, et probablement pour la même raison, les enseignants de FLE en Pologne y attachent une très grande importance, à tel point que de très nombreux apprenants finissent par croire que le subjonctif est à la fois le mode le plus français et particulièrement important du point de vue de l'efficacité de la communication réalisée en français. Les enseignants, surtout, semblent être responsables de cette représentation chez leurs disciples : «Les professeurs sont obsédés par le subjonctif, mais c'est le conditionnel qui paraît beaucoup plus important pour la communication de tous les jours et est plus fréquent »(R103). On peut supposer que la représentation sociale du subjonctif, partagée par beaucoup d'enseignants et d'apprenants polonophones, provoque partiellement ce sentiment de difficulté. Si l'on analyse les raisons pour lesquelles le subjonctif est considéré comme tellement difficile, deux, assez simples, se mettent au premier plan: la nécessité de mémoriser les formes irrégulières et les expressions après lesquelles s'utilise le subjonctif. Les paroles d'un des répondants pourraient résumer ce point : «Ce n'est pas difficile, mais il y a beaucoup d'expressions et il faut les connaître toutes, ne pas les oublier» (R31). Seulement trois répondants ont donné d'autres causes de sa difficulté : « le subjonctif parce qu'il n'est pas logique et régulier » (R22), « il manque de logique » (R21), « je ne le ressens pas » (R9). Quant à l'usage du subjonctif, mais aussi du conditionnel, la connaissance d'autres langues romanes (espagnol, italien), constitue, d'après quelques répondants, un obstacle, à cause des interférences.

Viennent ensuite, cette fois-ci dans les deux groupes d'étudiants, les articles (d'autres déterminants ne sont mentionnés que trois fois). Tous les répondants ont mis en avant le problème de la complexité des règles qui se superposent ou sont concurrentielles - les paroles d'un des répondants semblent illustrer le problème principal : «beaucoup de règles à retenir et (...) encore plus d'exceptions à ces règles » (R71). En même temps, durant l'analyse des résultats, les paroles de certains dévoilent leur sentiment de dichotomie entre l'envie de suivre les règles, apprises à l'école, et le recours à l'intuition dite langagière, acquise grâce aux expériences gagnées en dehors de l'école, p. ex. "Je les utilise intuitivement, mais je ne suis pas capable d'expliquer grammaticalement pourquoi j'ai utilisé tel ou tel article » (R8) ; «Il est plus facile d'utiliser les articles que de justifier leurs emplois» (R24); " Je ne sais jamais comment les utiliser: suivant les règles ou intuitivement, car ces deux approches s'excluent » (R29) ; «Il n'y a pas de règles précises pour chaque cas, de plus, différents livres de grammaire donnent différents exemples d'utilisation pour chaque type d'articles » (R44) ; "Hélas, les règles de leurs emplois ne vont pas de pair avec la réalité » (R45) ; «Je considère l'emploi des articles comme trop subjectif»(R84). Quelques répondants reprochent expressis verbis aux enseignants de langue/grammaire leur inaptitude à expliquer « la philosophie de l'article français » (R100) - bien que le domaine des articles ne se caractérise pas par l'univocité conceptuelle et que sa maitrise exige une longue pratique, se satisfaire de constater que "même les francophones ne les maîtrisent pas » (R98) ne peut pas être accepté ; il est fort probable que ces paroles incarnent l'une des représentations collectives les plus admises. Au lieu de donner les règles d'emploi des articles l'une après l'autre, il serait probablement plus pertinent de faire entrer les apprenants dans «la philosophie de l'article français », mentionnée ci-dessus, et de mettre ainsi l'accent sur ses valeurs sémantiques différentes; dans ce contexte, un des répondants met en exergue une difficulté majeure pour de nombreux apprenants polonais, ayant du mal à «définir quand une chose peut être considérée comme définie et déterminée » (R69). De plus, quelques futurs philologues ont attiré l'attention sur le manque de bonnes ressources d'apprentissage, y compris celles qui 
illustrent l'emploi des articles par l'intermédiaire de la traduction français-langue maternelle/langue maternelle-français ; cette approche comparative est fortement sollicitée.

$\mathrm{Au}$ total, 38 répondants ont aussi souligné leur difficulté à utiliser les temps du passé, principalement l'imparfait et le passé composé. Si les représentants du groupe A ont surtout évoqué le problème de superposition de l'usage dit intuitif et conforme aux règles $-\mathrm{p}$. ex. " Ils ne s'utilisent pas si intuitivement qu'il y paraît à première vue, il faut apprendre les règles » (R24) ou « Apparemment, leurs emplois sont faciles, mais en réalité, il y a pas mal de principes à garder, p. ex. utiliser le passé composé, si la durée est 'fermée' » (R53), les étudiants du groupe B voient plus de nuances de différentes natures, p. ex. « Parfois, j'ai du mal à déchiffrer un type de narration (...), ce qui m'empêche d'utiliser une forme correcte » (R83) ; «J'ai des difficultés à distinguer ce qui est 'descriptif' ou 'continu' de ce qui est 'ponctuel' et 'non continu' » (R90); "Dans tous les manuels de grammaire, on fait une distinction entre 'accompli' et 'non accompli', mais cette distinction est assez trompeuse ['zwodniczy' - notre traduction du polonais]: beaucoup d'idées sont exprimées à l'imparfait bien qu'elles concernent une réalité évidemment accomplie » (R101). Quant aux autres modes et temps français, les commentaires, particuliers, présentent un niveau plus élevé de généralité - en voici quelques exemples : «Il est plus facile de le reconnaître [passé simple] que de l'utiliser» (R42); «Le plus difficile est de créer une phrase au conditionnel, surtout le type si + plus-que-parfait + conditionnel passé » (R41) ; "Pour l'utiliser [discours indirect] correctement, il faut avoir en tête tout un système de temps et les utiliser en alternance, et c'est pourquoi on peut parfois se perdre » (R5).

L'usage des pronoms personnels, notamment pour ce qui est des compléments d'objet direct et indirect auprès des étudiants du groupe $\mathrm{A}$, constitue également un aspect difficile à maîtriser, et ceci pour quelques raisons : la méconnaissance de la construction des verbes, l'incapacité de distinguer le COD du COI, la nécessité de garder en mémoire un grand nombre de règles concurrentielles, provoquant «l'embarras du choix » aux yeux des répondants, la place et l'ordre des pronoms dans la phrase, plus que tout dans l'interrogation avec inversion. Il faudrait cependant ajouter que ce type de difficultés est annoncé surtout par les répondants du groupe A. Dans le groupe B, un autre obstacle se situe devant l'arrière-plan: «Il est facile d'utiliser des structures dans des exercices de grammaire, mais dans le langage parlé, quand il y a peu de temps pour réfléchir, cela devient problématique » (R59). C'est pourquoi les répondants requièrent l'augmentation du nombre d'activités visant l'automatisation de leurs emplois dans la conversation (spontanée, rapide, non préparée à l'avance, ...).

Par rapport au groupe A, les répondants du groupe B ont aussi mentionné les difficultés concernant l'accord du participe passé et la structure de la phrase française. Si le premier problème est plus facile à résoudre, car il est directement lié au besoin de mémoriser certaines règles et de garder une attention aiguë pendant l'écriture, le second résulte des interférences entre les langues française et polonaise - la structure de la phrase polonaise est plus souple et «s'adapte» aux besoins communicatifs et stylistiques (p. ex. les compléments peuvent être déplacés). Les répondants butent aussi contre les obstacles, s'ils sont obligés de choisir un articulateur logique approprié - dans ce contexte, les conjonctions de subordination provoquent le plus de malentendus. Pour ce qui est des deux groupes, d'autres difficultés énumérées dans le tableau 2 ont été signalées dans des cas particuliers et aucun commentaire approfondi n'a été fourni.

Pour achever notre analyse, il est nécessaire de dire que beaucoup de répondants des deux groupes ont mis l'accent sur une dissonance, particulièrement démotivante, entre leurs connaissances déclaratives en matière de grammaire française et leurs connaissances procédurales. Par conséquent, dans l'interaction, surtout l'interaction orale spontanée et imprévue, « il est impossible de garder la correction et de parler de manière fluide » (R39). Un des répondants accentue donc la nécessité de concilier la pratique à l'intuition - par 
rapport aux pronoms, il se prononce de la manière suivante : « (...) pour moi, il est question de les 'sentir', et de ne pas les comprendre » (R91).

\section{Conclusions}

Un regain d'intérêt pour la recherche concernant les perceptions subjectives est de plus en plus visible dans beaucoup de domaines. Saisissons le moment pour nous référer aux conclusions de l'essai cité dans la section 2.1, dans lesquelles C. Meyer (2001) rappelle les vertus de la collecte de données subjectives: "Nous savons, aujourd'hui, qu'un souci d'objectivité risque de conduire à une Science de l'Homme sans Homme. Notre mémoire collective a conservé le souvenir, encore vivace, de la visée hégémonique du structuralisme qui pensait, en empruntant la notion formelle de structure à l'algèbre, donner enfin une scientificité aux sciences humaines. Mais cette démarche a conduit à un savoir sans sujet ». L'idée d'étudier les représentations s'inscrit dans le postulat exprimé implicitement cidessus. D'une part, les recherches quantitatives dévoilent certaines tendances quant aux manières dont se manifestent la maîtrise de la langue et la conscience langagière ; d'autre part, les recherches qualitatives donnent accès à des explications plus nuancées des mécanismes cognitifs et affectifs mis en œuvre dans l'activité. Plus souvent qu'il ne peut y paraître, des facteurs affectifs et peu rationnels (craintes, croyances, idées reçues mécaniquement répétées, ...) agissent sur la perception de tous les sujets, y compris ceux en matière de grammaire.

D'après les résultats de notre étude empirique, trois facteurs prépondérants influencent la perception de la difficulté de la grammaire française : interférences entre la langue française et la langue maternelle, « concurrence » entre la volonté de se servir des règles de grammaire et le recours à l'intuition dite langagière (en réalité, difficilement définissable), idées reçues et stéréotypes portant sur la grammaire française. Beaucoup de difficultés ressenties par les répondants sont directement liées au caractère flou des sujets (temps du passé, choix des déterminants, choix des conjonctions de subordination), surtout ceux qui ne trouvent pas leurs correspondants dans la langue maternelle. Même si l'on peut éventuellement éprouver de la déception par rapport à la profondeur de certaines des réponses données, il faut supposer que ce sont les représentations mentales et les idées reçues envers le degré de difficulté qui stimulent le degré de difficulté ressenti. En réalité, parfois, aucun argument rationnel n'explique le succès de certaines opinions et un autre danger surgit à l'horizon : le processus de stéréotypisation peut être étayé par les enseignants de langue/grammaire. Les représentations sociales, donc partagées par des groupes sociaux plus vastes, en endossent également la responsabilité.

Dans l'avenir, des recherches plus sophistiquées sont à continuer et à creuser dans ce domaine, et les thèmes se distinguant par leur fécondité ne manquent pas (représentations de soi-même en tant qu'apprenant en grammaire, représentations de différentes façons d'acquérir la grammaire, manières de conceptualiser certains aspects grammaticaux, etc.). Mais indépendamment du sujet de l'étude, il nous semble important d'accentuer la nécessité de diversifier les techniques de collecte de données et de les analyser aussi bien dans une perspective transversale que longitudinale pour voir leur évolution. Contrairement à certaines opinions (souvent diffusées par les médias), simplifiant le problème, l'acquisition de la langue, donc tout particulièrement de la grammaire, ne se fait jamais involontairement et spontanément, et les représentations envers ce processus agissent fortement sur celle et celui qui est en train de l'acquérir. Leur fardeau est quelquefois très lourd à porter, mais ... trop méconnu pour l'affronter. 


\section{Références bibliographiques}

Castelotti, V. (dir.). (2001). D'une langue à d'autres: pratiques et représentations. Rouen: Publications de l'Université de Rouen.

Castelotti, V. et Moore, D. (2002). Représentations sociales des langues et enseignements. Guide pour l'élaboration des politiques linguistiques éducatives en Europe - De la diversité linguistique à l'éducation plurilingue. Strasbourg: Conseil de l'Europe, Division des politiques linguistiques.

Corder, S.P. (1973). The elicitation of interlanguage. [in] Svartvik, J. (dir.). Errata. Papers in Error Analysis, Lund : Gleerup, 36-48.

Cuq, J.-P. et Gruca, I. (2005). Cours de didactique du français langue étrangère et seconde. Grenoble : PUG.

Durkheim, É. (1898). Représentations individuelles et représentations collectives. Revue de Métaphysique et de Morale, VI.

Meyer, C. (2001). Les représentations mentales. Entre «res» et «flatus vocis». Communication, 21/1, 9-31.

Moscovici, S. (1961). La psychanalyse, son image et son public. Paris : PUF.

Schopenhauer, A. (1818/2004). Le monde comme volonté et comme représentation. Paris : PUF.

Smuk, M. (2017). Construire son identité dans les récits autonarratifs - cas des apprenants en langue. Bulletin suisse de linguistique appliquée (VALS-ASLA), numéro spécial, 195-204.

Vasseur, M.-T. et Arditty, J. (1996). Les activités réflexives en situation de communication exolingue : réflexions sur quinze ans de recherche. Acquisition et interaction en langue étrangère, 8, 57-87.

www.lmg.ulg.ac.be/competences/chantier/methodo/meth_repres.html 